\title{
A weekly speech and language therapy service for head and neck radiotherapy patients during treatment: maximizing accessibility and efficiency
}

\author{
Louise C Occomore, MSc, and Zoë Knight, MSc \\ Speech and Language Therapy Department, Barts Health NHS Trust, St Bartholomew's Hospital, West Smithfield, London, United \\ Kingdom
}

\begin{abstract}
Background Our hospital did not provide a weekly speech and language therapy (SLT) service for head and neck cancer patients during radiotherapy treatment. SLT is recommended in the international guidelines, but many centers do not offer this service. In the case of our hospital, SLT was not provided because there were no funds to cover the costs of additional staff.

Objectives To create a new service model within a multidisciplinary setting to comply with the international SLT guidelines and without increasing staff. We aimed to measure the accessibility and efficiency of a new model of service delivery at our center both for patients and for the service.

Methods 79 patients were recruited for the study. We followed 1 group of patients ( $n=29$; observation group) throughout their treatment for 6 weeks to establish if there was a clinical need to offer SLT at the treatment center. A second group of patients ( $\mathrm{n}=$ 50; intervention group) received a weekly SLT review at the treatment center throughout their radiotherapy. Data collected at the tertiary cancer center for 6 months included: age, gender, tumor site and size, treatment modality, swallowing outcomes, communication outcomes, patient satisfaction, multidisciplinary team feedback, and time efficiency. The observation group did not participate in the intervention group because the data was collected between 2 different groups of participants. However, all participants were referred to their local SLT service at the end of their treatment if that was clinically indicated, regardless of the group they had been in.

Results The proportion of patients accessing SLT services during treatment and the time efficiency of the service were both improved with this model of delivery. The service's compliance with international guidelines was met. More patients continued with oral intake during their treatment at our center with the new service. Improvements were also reported in communication clarity and communication confidence in the same group.

Conclusion Offering head and neck cancer patients SLT at the same time and place as their radiotherapy treatment improves patient outcomes and increases SLT efficiencies. As this was not a treatment study, further clinical trials are required with regards to functional outcomes.
\end{abstract}

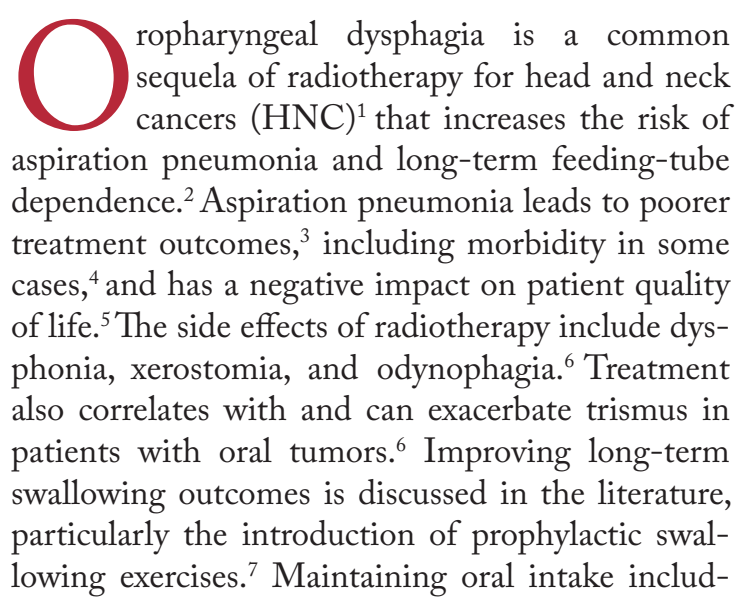

ing maximising textures during treatment to avoid dependence on alternative feeding, ${ }^{8,9}$ along with treatment advances such as intensity-modulated radiotherapy (IMRT), ${ }^{10}$ improves long-term swallowing outcomes.

Current international guidelines recommend that patients with HNC should start SLT before treatments that may result in swallowing and/or communication difficulties. ${ }^{11,12}$ Patients should be seen regularly during radiotherapy at the treatment center, ${ }^{11}$ with "particular attention to speech and swallowing" for sites such as the hypopharynx. ${ }^{12}$

Since the publication of NICE guidelines more than a decade ago, evidence for SLT intervention

Accepted for publication May 1, 2015. Correspondence: Louise C Occomore, MSc; louise.occomore@nhs.net. Disclosures: The authors have no disclosures. JCSO 2015;13:248-255. @2015 Frontline Medical Communications. DOI 10.12788/ icso.0149. 
in the HNC patient population has increased. ${ }^{8,7}$ There is a strong focus on functional swallowing outcomes, which is a significant but not exhaustive aspect of SLT's role with this patient population. SLT also has a role in the management of communication impairments, stoma and voice prosthesis management in laryngectomees, and trismus management, among others.

A survey done in the United Kingdom found that 69\% of SLT teams offer on-treatment services to patients, with $25.6 \%$ offering weekly input. ${ }^{13}$ A study of speech and language therapists in the United States found that only 18.3\% of respondents intervened proactively with patients who were undergoing radiotherapy ${ }^{14}$ and that there was poor consensus among them about what on-treatment therapy should entail. Kiss et $\mathrm{al}^{15}$ found that proactive dietetics intervention during and after radiotherapy reduced hospital admissions and unplanned nasogastric tube insertions; however, there is a lack of similar findings demonstrating the positive contributions of SLT to patient outcomes.

At our institution, patients travelled as much as a 50 -mile round trip daily to receive radiotherapy treatment for up to 7 weeks. Before we established an SLT service at the treatment center, many patients underwent radiotherapy for $\mathrm{HNC}$ without access to SLT. Those who were offered the standard SLT intervention at their local hospitals were often too fatigued to attend SLT in addition to coming to the center for their treatment. As with many other centers across the UK and abroad, and based on the authors' experiences, the SLT service was often reactive. Many patients were referred to SLT weeks or months after treatment initiation with severe, chronic effects (eg, trismus, oropharyngeal dysphagia, and long-term alternative feeding dependence).

We were unsuccessful at our center in our efforts to get more SLT resources for patients who were undergoing radiotherapy for HNC, which was a reflection of the current financial constraints within the British National Health Service. We learned in discussions with our clinical colleagues at other centers that they had encountered similar financial barriers. As a result, we wanted to start a weekly SLT service for this patient population at our center. We wanted to determine if the weekly SLT intervention would improve patients' access to a speech and language therapist and whether we could provide the service without increasing staff.

\section{Methods}

We recruited 79 patients who were divided into 2 groups the observation group $(\mathrm{n}=29)$ and the intervention group $(n=50)$. The data was collected within the context of the speech and language therapist's clinical work and was not a formal research study, so ethical approval was not required in agreement with the center's Research Lead and Clinical Effectiveness Unit.

\section{Observation group}

A group of 29 patients received standard treatment during radiotherapy. Standard treatment consisted of no SLT intervention at the treatment center during radiotherapy. Some patients had received SLT previously in their treatment pathway (eg, when they had already undergone surgery and were having adjuvant treatment). Others had no SLT throughout their care. We therefore asked the subjects in the observation group whether or not they had ever seen an SLT before their current radiotherapy treatment as part of the data collection process.

Before we offered the new SLT service, we had to establish if there was a need for the service. We used the data from the observation group as a baseline of patients' access to SLT and their functional outcomes at the end of radiotherapy. The data were collected weekly over 6 weeks. All of the patients were undergoing radiotherapy for diagnosed HNC. Some patients (Table 1) also received concurrent chemotherapy, which increases the severity of radiotherapy side effects. ${ }^{16}$ All of the patients who were undergoing radiotherapy to the head and neck at the treatment center over a period of 6 weeks were included in the study. There were no exclusion criteria.

\section{Intervention group}

A group of 50 patients was offered weekly SLT intervention at the treatment center. Data were collected over 6 months. The patients were undergoing radiotherapy to the head and neck (with or without chemotherapy). There were no exclusion criteria. None of the patients declined treatment.

One speech and language therapist was based in the radiotherapy department 1 day a week alongside the multidisciplinary team (MDT) to collect data from both groups. The MDT included clinical oncologists, a dietitian, clinical nurse specialists, radiotherapy nurses, and a radiographer, all of whom had specialist skills in head and neck oncology. All of the patients were seen weekly by the MDT during treatment.

\section{Data collection}

Triage of clinical need. The therapist attended the weekly MDT clinic for both groups of patients. With patients' consent, the therapist was also present for each of their weekly oncology consultations as part of the data collection. The oncologist monitored patients' acute morbidities during each consultation through open questioning and the use of a locally developed toxicities monitoring form. This was adapted from relevant sections from the Common Terminology Criteria for Adverse Events. ${ }^{17}$ By observing these consultations, the therapist was able to triage each patient to determine if they presented with any specific SLT needs (eg, dysphonia, dysphagia, trismus, and 


\section{Original Report}

neck stoma care). The therapist recorded these symptoms for both groups to determine if there was a justifiable need for SLT in the MDT clinic; and also to inform the type of SLT treatment required for the intervention group. The following data were collected from both groups: age, gender, tumor site and size, treatment modality (radiotherapy with or without chemotherapy), and treatment dose.

Speech and language therapy outcome measure (SALTOM). Patients in both groups were asked to complete the SALTOM at the end of their treatment. The SALTOM $^{18}$ is an outcome measure based on questions specifically related to communication, voice, and swallowing function. It has 11 questions about communication, and 5 about swallowing. All of the questions are closed, with a combination of Yes/No and visual analogue scale responses. This is a tool used at our centre containing questions relating to all domains of speech, voice and swallowing. It is not standardised but is used in the absence of a similar standardised outcome tool across all of these domains in one document. The authors note here that the current paper is not a treatment study, however, as data was collected as part of our clinical work, we collected clinical outcome data in accordance with best practice, as is standard care at our center. The data that were collected demonstrated the level of clinical need in this population at our center.

Patient satisfaction survey. Patients in the intervention group were asked to complete a satisfaction survey at the end of their treatment. We developed this survey at our center, and it was approved by the Information Governance department at St Bartholomew's Hospital (available on request). This was given to the intervention group only because the observation group did not receive any SLT intervention at the treatment center. The form contained 4 closed and 2 open questions relating to patients' satisfaction with the service they received.

Time efficiency and accessibility. We collected the following data:

- At the time of triage, we asked patients in both groups whether they had ever seen a speech and language therapist either before or during their treatment, and whether it was at the treatment center or at their local hospital.

- If patients reported they had seen a therapist, we asked them if it was a weekly contact during their radiotherapy treatment as per NICE guidelines.

- The standard service model was used before the implementation of the new service and did not include the patient seeing a speech and language therapist at the treatment center during radiotherapy. The standard service model allowed for patients to see a therapist at their local hospital. We calculated the average number of

\begin{tabular}{|c|c|c|}
\hline \multirow[b]{3}{*}{ Age range, y (mean) } & \multicolumn{2}{|c|}{ Group, n (\%) } \\
\hline & $\begin{array}{c}\text { Observation } \\
(\mathbf{n}=29)\end{array}$ & $\begin{array}{c}\text { Intervention } \\
(\mathbf{n}=50)\end{array}$ \\
\hline & $33-88(57)$ & $38-85(61)$ \\
\hline Men & $22(76)$ & $36(72)$ \\
\hline Women & $7(24)$ & $14(28)$ \\
\hline \multicolumn{3}{|l|}{ Cancer type } \\
\hline $\begin{array}{l}\text { Nasopharyngeal } \\
\text { primary }\end{array}$ & $2(6.9)$ & 0 \\
\hline Oral cavity primary & $6(20.7)$ & $13(26)$ \\
\hline Oropharyngeal & $12(41.4)$ & $17(34)$ \\
\hline Hypopharyngeal & 0 & $3(6)$ \\
\hline Laryngeal & $7(24.1)$ & $12(24)$ \\
\hline $\begin{array}{l}\text { Unknown primary } \\
\text { (neck node) }\end{array}$ & $1(3.4)$ & $3(6)$ \\
\hline Lymphoma (neck node) & $1(3.4)$ & 0 \\
\hline Pinna & 0 & $2(4)$ \\
\hline \multicolumn{3}{|l|}{ Stage } \\
\hline TO/X & $2(6.9)$ & $7(14)$ \\
\hline $\mathrm{T} 1$ & $2(6.9)$ & $9(18)$ \\
\hline $\mathrm{T} 2$ & $7(24.1)$ & $12(24)$ \\
\hline T3 & $6(20.7)$ & $10(20)$ \\
\hline $\mathrm{T} 4$ & $9(31)$ & $10(20)$ \\
\hline Other/unstaged & $2(6.9)$ & $2(4)$ \\
\hline \multicolumn{3}{|l|}{ Type of therapy } \\
\hline Radiotx alone & $11(38)$ & $27(64)$ \\
\hline Chemo-radiotx & $18(62)$ & $23(46)$ \\
\hline Primary treatment & $11(38)$ & $17(34)$ \\
\hline $\begin{array}{l}\text { Adjuvant treatment } \\
\text { after surgery }\end{array}$ & $18(62)$ & $33(66))$ \\
\hline Radical radiotx dose & $28(97)$ & $48(96)$ \\
\hline Palliative radiotx dose & $1(3)$ & $2(4)$ \\
\hline
\end{tabular}

patients seen in a standard 7.5 hour day in our standard model of outpatient service delivery. We then calculated our average patient nonattendance rate in the standard outpatient model.

- The new service model included weekly SLT for all patients who were undergoing radiotherapy at the treatment center in the MDT setting. We calculated the same data for the new SLT service model from the intervention group; the average number of patients seen in a 7.5 hour day, and the nonattendance rate. 


\section{Intervention}

We have already noted that this paper outlines a new model of service delivery and is not a treatment study. The results relate to offering a weekly SLT service at the treatment center during radiotherapy. This service was previously not offered to patients, and we therefore aimed to establish the efficiency and accessibility of providing this service. The study does not investigate the clinical effectiveness of a particular therapy approach used by SLTs with this caseload.

The SLT intervention was individualized according to the patient's clinical need. The various types of interventions included: removing a voice prosthesis to allow a route for tracheoesophageal tube feeding in the event of structural aphagia in a laryngectomee; teaching swallowing exercises; training in the use of passive jaw stretching devices; and counselling and support around communication difficulties, among others. Patient feedback and reported outcomes were not related to a specific intervention or treatment because of the heterogeneity of the patient group and the patients' clinical needs.

\section{Multidisciplinary team feedback}

We requested qualitative feedback about the new service from the MDT members after 6 months of providing the new service. The team included 2 consulting clinical oncologists; 2 specialist registrars; a head and neck radiographer; and a head and neck dietitian. We also asked 5 communitybased speech and language therapists who before the new service would have treated some patients at their local hospitals during their radiotherapy in the standard treatment model. The new service was likely to have an impact on their caseloads and referral rates, and they had experience with the previous model of service delivery. We e-mailed each of the team members to ask for their views on the new service, specifically: the impact of the new service on patients, and its impact on the MDT treatment pathway. No patient outcome data was given to the professionals until after we received their feedback.

\section{Results}

We aimed to increase compliance with international guidelines for SLT in HNC patients by reviewing patients weekly at our treatment center during their radiotherapy and without increasing staff. We wanted to determine if we could do that without compromising patient outcomes at our own center, and to elicit patients' and professionals' opinions on the new model of service delivery. We wanted to investigate if that would improve patients' access to SLT during treatment and time efficiency for our service by treating them in an MDT setting. Results were analyzed with descriptive statistics (Table 1 ). The typical radical radiotherapy treatment regimens given at our center were either 66 Gray in 33 fractions, or 70 Gray in 35 fractions.

\section{Triage of clinical need}

The speech and language therapist identified a clinical need for an SLT intervention in $76 \%$ of patients who were triaged. The specific type of disorder or clinical need was recorded (Figure 1).

\section{Outcome measures}

The SALTOM return rate was $31 \%$ in the observation group, and $36 \%$ in the intervention group. Only $30 \%$ of the forms disseminated to the intervention group were completed sufficiently to analyze. The patient-reported functional outcomes for both groups for swallowing are shown in Table 2 and for communication, in Table 3.

\section{Patient satisfaction survey}

The return rate for the intervention group was 30\%. The form was not given to patients in the observation group because they did not receive a service at the center. Patients indicated that the SLT was informative and helpful, supportive and easy to access, provided them with useful advice, and should be provided at least weekly. In all, 75\% of the patients said the treatment had been helpful, and $25 \%$ did not respond.

\section{Time efficiency and accessibility}

A total of $44 \%$ of patients in the observation group reported having seen a speech and language therapist at some point during their treatment pathway prior to their radiotherapy. No patients in either of the groups had been seen weekly during their treatment by a therapist. All of the patients in the intervention group were seen weekly by a speech and language therapist who followed the guidelines of the new model of service delivery.

With the standard outpatient model of service delivery at our center, the observation group was offered a referral to SLT. One therapist was able to offer 6 outpatient appointments per working day. The average non-attendance rate at these appointments was $12 \%$.

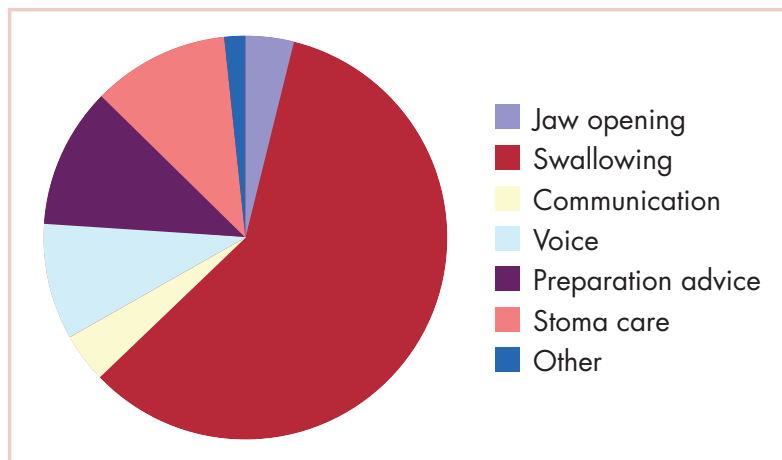

FIGURE 1 Patients' speech and language therapy needs on presentation in the radiotherapy clinic. 


\begin{tabular}{|c|c|c|}
\hline \multirow[b]{2}{*}{ Outcome } & \multicolumn{2}{|c|}{ Group, n (\%) } \\
\hline & $\begin{array}{l}\text { Observation } \\
(\mathbf{n}=\mathbf{2 9})\end{array}$ & $\begin{array}{l}\text { Intervention } \\
\quad(\mathbf{n}=\mathbf{5 0})\end{array}$ \\
\hline $\begin{array}{l}\text { Continuing to take oral } \\
\text { fluids }\end{array}$ & $6(66.6)$ & $10^{\circ}(90.9)$ \\
\hline Continuing to take oral diet & $5(55.5)$ & $7^{b}(63.6)$ \\
\hline Taking a pureed diet & $2(33.3)$ & $2(28.5)$ \\
\hline Taking a soft diet & $3(50.1)$ & $3(42.9)$ \\
\hline $\begin{array}{l}\text { Taking a modified normal } \\
\text { diet }\end{array}$ & $1(16.6)$ & $2(28.6)$ \\
\hline Taking a normal diet & 0 & 0 \\
\hline $\begin{array}{l}\text { Patients with little or no } \\
\text { concern about eating and } \\
\text { drinking in front of others }\end{array}$ & $4(44)$ & $8(72.8)$ \\
\hline \multicolumn{3}{|c|}{$\begin{array}{l}\text { In one case, the patient reported being unable to manage thin drinks and } \\
\text { required thickened fluids. }\end{array}$} \\
\hline \multicolumn{3}{|c|}{$\begin{array}{l}\text { bTwo patients stated specifically that they could not manage normal solids, bu } \\
\text { could manage soft and pureed foods respectively. }\end{array}$} \\
\hline
\end{tabular}

\begin{tabular}{|c|c|c|}
\hline \multirow[b]{2}{*}{ Outcome } & \multicolumn{2}{|c|}{ Group, n (\%) } \\
\hline & $\begin{array}{l}\text { Observation } \\
(\mathbf{n}=29)\end{array}$ & $\begin{array}{l}\text { Intervention } \\
(\mathrm{n}=50)\end{array}$ \\
\hline \multicolumn{3}{|l|}{ Voice quality } \\
\hline Good/very good & $1(11.1)$ & $3(27.3)$ \\
\hline Fair & $4(44)$ & $4(36.4)$ \\
\hline Poor/very poor & $4(44)$ & $4(36.4)$ \\
\hline \multicolumn{3}{|l|}{ Communication clarity } \\
\hline Good/very good & $1(11.1)$ & $4(36.4)$ \\
\hline Fair & $5(55.6)$ & $4(36.4)$ \\
\hline Poor/very poor & $3(33.3)$ & $3(27.3)$ \\
\hline
\end{tabular}

In the new model of service delivery provided for the intervention group, 1 therapist was able to offer 12 appointments with no consultations unattended within the MDT setting.

\section{Intervention}

As already discussed, this is not an intervention study; however, Figure 1 shows the nature of the diagnoses and disorders with which patients presented in the weekly clinic. This demonstrates the variety of the treatments provided and therefore the breadth of knowledge and skills that a speech and language therapist requires when working with this population in this service model.

\section{MDT feedback}

Feedback from the MDT stated that weekly SLT for patients during radiotherapy helped to:

- Assess and provide support to patients during their treatment, as needed and with a proactive approach to problem-solving;

- Provide a smoother care pathway for patients undergoing treatment;

- Maintain the use of mouth bites throughout patient treatment through timely trismus intervention (the mouth bite is a device is worn during radiotherapy to maximise the precision of treatment by maintaining a static tongue position for lateral tongue tumors or maintaining the distance between the mandible and maxilla); and

- Enhance knowledge of the MDT within the clinic.

\section{Discussion}

We started a new weekly on-treatment service for a group of patients who were undergoing radiotherapy for head and neck cancer. We provided the service within an MDT setting and at the site of treatment to determine if it would improve accessibility for patients, improve attendance, and enhance outcomes at our center. We also aimed to establish if we could provide the new service without an increase in staff or clinical time.

The results showed that $76 \%$ of patients presented in the weekly MDT clinic with a clinical need for SLT. By triaging patients during a 10 -minute consultation with their oncologist, we found it was possible to determine which patients required direct SLT intervention. We did not intervene directly with $24 \%$ of patients after their triage session. However, the 10 minutes spent gathering relevant triage information provided an opportunity for the speech and language therapist to introduce the patient to the role of SLT and to outline some of the symptoms that may require SLT later on in their treatment. This provided patients with education and equipped them to have more control over their health care needs by raising their awareness of the symptoms they needed to watch for and what to expect at each stage of treatment. It also provided an opportunity for the therapist to demonstrate to the rest of the MDT which patients would or would not benefit from the SLT intervention, and why. This was reflected in the MDT feedback in which team members noted that they had an enhanced understanding through working directly with the therapist. Over time, the authors have experienced that this MDT model improves the understanding of our role and is reflected in the referrals we receive as a team.

The current paper does not outline an intervention study. The heterogeneity of patients, and therefore the interventions provided were far reaching (Figure 1, Table 1). Our functional outcome measures collected routinely at the 
end of the patients' treatment should be generalized with caution to other centers and patients. The patients' selfreported outcome measure forms showed that $23 \%$ more patients in the intervention group compared with the observation group were taking oral fluids at the end of their treatment, which resulted in fewer patients being nil by mouth. Maintaining oral intake throughout treatment has been shown to improve long-term swallowing outcomes, ${ }^{8,9}$ so it is conceivable that weekly SLT intervention during the patients' radiotherapy may have a role in improving long-term swallowing outcomes.

Further studies are needed to establish if there is a treatment effect specifically related to our model of service delivery. More patients in the intervention group than in the observation group continued to take oral solids at the end of their treatment. Side effects (eg, oedema, mucositis, odynophagia, dysguesia, xerostomia, and trismus) are more likely to cause dysphagia to solids than to liquids, which may explain the smaller difference between the groups for solids. Among patients who continued with oral solids to the end of their treatment, the range of textures they were able to manage was greater in the intervention group than in the observation group. Clinical trials are also needed to establish the impact of SLT intervention on patients' functional outcomes at the end of treatment and how that has an impact on their long-term function.

Patients in the intervention group rated their communication clarity and voice quality as superior to those in the observation group. This was an unexpected finding as there is no evidence to the authors' knowledge that SLT intervention reduces dysphonia or dysarthria during treatment. Vocal hygiene advice was offered to all patients who presented with dysphonia during treatment, and advice about speech clarity was given to patients with dysarthria, along with counselling and reassurance. It is not clear if the improved ratings in the intervention group were owing to improved function or reduced psychosocial impact through education and counselling. This may be reflected in the patients' feedback that receiving a weekly SLT intervention at the treatment center during their radiotherapy was informative, supportive, and provided useful advice. The importance of education, ${ }^{19}$ problem-solving during treatment, ${ }^{20}$ and their impact on psychosocial well-being, ${ }^{20,21}$ has been well documented. More robust evidence is required to demonstrate the effect of SLT intervention on voice and speech parameters during treatment.

Within the British National Health Service, there is a drive to provide high-quality, evidence-based care in the face of limited resources and that demands time and costefficient models of service delivery that meet national guidelines. A significant barrier to our service in providing a weekly on-treatment service to all HNC radiotherapy patients was the recognition that this would increase the number of patients who accessed our service. The observation group data show that less than half of patients (44\%) had ever seen a speech and language therapist during their treatment pathway, and this was not weekly during their treatment. We therefore predicted at least double the number of consultations being required on a weekly basis. Within our standard model of service delivery, this would require us to employ more staff, and business cases for increased funding were unsuccessful. We wanted to establish whether changing our model of service delivery would allow us to provide a weekly review service to all patients without increasing our staff. In our existing model of service delivery, we also had a nonattendance rate of $12 \%$, which placed further pressure on services.

We found that by providing weekly proactive SLT intervention at the treatment center during radiotherapy, we were able to increase the percentage of patients who accessed our service from $44 \%$ to $100 \%$. This was achieved by offering the service and an MDT assessment within the radiotherapy department at time of treatment. Our service was more convenient for patients both in timing and location. In the authors' experience, it is often the patients who are at high clinical risk who do not attend separate outpatient appointments, because those who are close to the end of their treatment and are experiencing high toxicity are at a greater risk for dysphagia and trismus, for example. Therefore, the service we were providing with our old model was least accessible to the most vulnerable of patients.

We also find in our clinical experience that patients may not be clear about the purpose of SLT appointments in outpatient clinics, and that may contribute to their nonattendance. For example, when asking patients about their nonattendance in SLT clinics, they sometimes report that they have no speech concerns and were not aware that a speech and language therapist might have helped with their swallowing difficulties. In our new model of service delivery, patients reported a symptom such as trismus to their oncologist, and the therapist was immediately able to explain how an assessment and some therapy might benefit the patient before offering them a separate consultation straight away. In addition to providing the intervention in a timely manner and at a convenient location, the value of contact with the therapist is made clear to the patient in the context of their own concerns and symptoms.

We found that the new model of service delivery halved the average contact length with the patient from 60 to 30 minutes. This was achieved by triaging the patients after gathering information about any symptoms they were experiencing each week and deciding which of those might require or respond to SLT treatment. In our standard treatment model, we would have allowed for a 60 -minute outpatient appointment for each patient, which we now know is not always necessary based on our findings in the inter- 
vention group. By providing the service based on the new model, we were able offer 10 minutes to a patient where only a short contact with them was required, but we were able to see other patients for 60 minutes if it was clinically indicated. Because the patients were already in the department and had appointments with various MDT professionals as well as receiving their treatment, we were able to be more flexible by allotting patients the SLT times around their other appointments. We also found that the new model of service delivery facilitated a closer working relationship between the SLT team and their MDT colleagues, which allowed for more timely communication between them. With the standard outpatient model, the therapist would need to spend part of the allocated 60 minutes e-mailing colleagues, writing reports, or making telephone calls to ensure that all parties were in agreement with the management plans. We found that this time was reduced by seeing patients within the Radiotherapy department alongside our MDT colleagues on the same day of the week, as we were able to initiate these discussions face-to-face, rather than awaiting a response to e-mail for example.

By seeing patients weekly as part of a proactive model of service delivery, symptoms can also be addressed as they arise and patients can be prepared in advance to reduce the need for prolonged reactive problem-solving. Where prophylactic interventions such as jaw stretches, diet modification ideas, and swallowing exercises can be provided in advance of symptoms occurring, patients can be more empowered to be involved in their own care before problems become severe and the patient has to be placed on an SLT waiting list to receive treatment. Although the number of patients who accessed our service more than doubled, we were able to meet the needs of our patients within international guidelines and without additional time or staff by making changes to our service delivery model.

Members of the MDT reported that all of the patients in the intervention group continued to use their mouth bites comfortably throughout the course of their treatment, which had been unusual before the new service was offered. This was an unexpected finding. Further clinical trials on SLT intervention for trismus during treatment are required because our sample size was too small to confirm a treatment effect.

\section{Limitations}

Data was collected as part of our routine clinical work at the center with a view to starting the new SLT on-treatment service. The previous absence of such a service presented an opportunity to record baseline data from an observation group who did not receive weekly SLT intervention at the treatment center. However, because it was not a formal research study, we were not able to match groups or use inclusion and exclusion criteria. Collection of baseline data was necessary in establishing whether there was a clinical justification for the new service. After the new service had been initiated, no patients could ethically be excluded from receiving the weekly intervention. Patients were recruited consecutively, and as such, no biases were present in the data in this regard. Data was collected from the observation group for only 6 weeks compared with 6 months of data from the intervention group. As discussed, this was due to the data being taken from clinical work, whereby once we had justified a need for our intervention it was ethical to begin providing this as soon as possible.

The low return rate for the feedback form and the SALTOM affected our ability to generalize these findings to other patients and treatment settings. The factors identified as contributing to the low return rate were: lack of an interpreter; patient illiteracy; stopping treatment early; and the therapist not being able to contact the patient during the final treatment.

We did not conduct a statistical analysis of the data because of the small number of patients in the study. It is not unusual to face difficulties when trying to engage the $\mathrm{HNC}$ population in research because of the epidemiological nature of the disease. HNC correlates with low education and high illiteracy levels, and high rates of alcohol consumption. ${ }^{22}$ Patients often present late with advanced disease. Our center serves 2 of the lowest socioeconomic boroughs in London, with some of the highest poverty rates in the UK. ${ }^{23}$ In addition, it has high rates of homelessness, unemployment, and premature mortality. For patients who have been through difficult and tiring treatment such as radiotherapy, it is not surprising that the survey return rate was low given the context in which we work.

\section{Conclusion}

We aimed to establish whether weekly SLT intervention during patients' radiotherapy treatment at our treatment center would improve access to SLT and provide a more time-efficient model of service delivery while still achieving good clinical outcomes and patient satisfaction. This was driven by international guidelines for best practice.

Our data shows that we were able to provide a weekly on-treatment review service for all patients who were undergoing radiotherapy for head and neck cancer without increasing clinical time or staffing. We provided this without compromising patient satisfaction or patient outcomes. We found improved functional outcomes for those patients who received interventions based on the new model of service delivery, though we emphasize that our results do not lend themselves to generalization. We achieved those improved outcomes by directing the most time and intervention to those patients for whom it was clinically indicated through triage; working more closely with the MDT to reduce remote liaison time; and eliminating nonatten- 
dance. By triaging patients and assessing them at a time and setting that was convenient for them, we were able to identify and access those who were most at risk. For patients who were identified as being at low risk, we eliminated a short or unnecessary consultation in an outpatient clinic, and empowered them to monitor their own symptoms and contact our department. The quality of the intervention was not impacted, even though the average contact time was halved. Patients and the MDT reported that they were satisfied with the new model of service delivery.

Further research is required to establish the long-term impact of this service for patients (ie, duration of tube feeding after treatment) and long-term functional outcomes. A larger cohort of patients is needed to investigate functional outcomes more robustly, and the impact of SLT during treatment on other SLT-related diagnoses such as communication disorders and trismus.

\section{Acknowledgments}

The authors acknowledge Dr Melanie Powell, Dr Justin Roe, Dr Deanna Gibbs, Dr Harriet Shannon, and Carol Stokes for their advice and encouragement in preparing this manuscript. They also thank Dr Amen Sibtain, Dr Melanie Powell, and the MDT for their support in initiating and developing the new SLT service in our center's radiotherapy department. The authors are grateful to the patients at our center for their participation in and support for the study.

\section{References}

1. Platteaux N, Dirix P, Dejaeger E, Nuyts S. Dysphagia in head and neck cancer patients treated with chemoradiotherapy. Dysphagia. 2010;25:139-152.

2. Bleier BS, Levine, MS, Mick R, et al. Dysphagia after chemoradiation: analysis by modified barium swallow. Ann Otol Rhinol Laryngol. 2007;116:837-841.

3. Chen SW, Yang SN, Liang JA, Lin FJ. The outcome and prognostic factors in patients with aspiration pneumonia during concurrent chemoradiotherapy for head and neck cancer. Eur J Cancer Care. 2010;19:631-635.

4. Mortensen HR, Jensen K, Grau C. Aspiration pneumonia in patients treated with radiotherapy for head and neck cancer. Acta Oncol. 2013;52:270-276.

5. Nguyen NP, Frank C, Moltz CC, et al. Impact of dysphagia on quality of life after treatment of head-and-neck cancer. Int J Radiat Oncol Biol Phys. 2005;61:772-778.

6. Vissink A, Jansma J, Spijkervet FK, Burlage FR, Coppes RP. Oral sequelae of head and neck radiotherapy. Crit Rev Oral Biol Med. 2003;14:199-212.

7. Roe JW, Ashforth KM. Prophylactic swallowing exercises for patients receiving radiotherapy for head and neck cancer. Curr Opin Otolaryngol Head Neck Surg. 2011;19:144-149.

8. Hutcheson KA, Bhayani MK, Beadle BM, et al. Eat and exer- cise during radiotherapy or chemoradiotherapy for pharyngeal cancers: use it or lose it. JAMA Otolaryngol Head Neck Surg. 2013;139:1127-1134.

9. Langmore S, Krisciunas GP, Miloro KV, Evans SR, Cheng DM. Does PEG use cause dysphagia in head and neck cancer patients? Dysphagia. 2012;27:251-259.

10. Peponi E, Glanzmann C, Willi B, Huber G, Studer G. Dysphagia in head and neck cancer patients following intensity modulated radiotherapy (IMRT). Radiat Oncol. 2011;6:1. DOI:10.1186/1748717X-6-1.

11. Guidance on cancer services: improving outcomes in head and neck cancer. National Institute for Clinical Excellence. https://www.nice. org.uk/guidance/csghn/evidence/improving-outcomes-in-head-andneck-cancers-the-manual2. Published November 2004. Accessed July 12, 2015.

12. NCCN Clinical Practice Guidelines in Oncology (NCCN Guidelines), head and neck cancers. National Comprehensive Cancer Network. Version 2.2013. http://www.nccn.org/professionals/physician gls/f_guidelines.asp\#site. Published 2013. Accessed July 12, 2015.

13. Roe JW, Carding PN, Rhys-Evans PH, Newbold KL, Harrington HJ, Nutting CM. Assessment and management of dysphagia in patients with head and neck cancer who receive radiotherapy in the United Kingdom - a web-based survey. Oral Oncol. 2012;48:343348.

14. Krisciunas GP, Sokoloff W, Stepas K, Langmore SE. Survey of usual practice: dysphagia therapy in head and neck cancer patients. Dysphagia. 2012;27:538-549.

15. Kiss NK, Krishnasamy M, Loeliger J, Granados A, Dutu G, Corry J A dietitian-led clinic for patients receiving (chemo) radiotherapy for head and neck cancer. Support Care Cancer. 2012;20:2111-2120.

16. Wilson JA, Carding PN, Patterson JM. Dysphagia after nonsurgical head and neck cancer treatment: patients' perspectives. Otolaryngol Head Neck Surg. 2011;145:767-771.

17. Common Terminology Criteria for Adverse Events (CTCAE). National Cancer Institute. http://evs.nci.nih.gov/ftp1/CTCAE/CTCAE_4.03_2010-06-14_QuickReference_8.5x11.pdf. Last updated June 14, 2010. Accessed July 12, 2015.

18. Knight, $Z$. The development and validation of a self rating questionnaire to measure the outcome of speech and language therapy intervention in the head and neck cancer population. Paper presented at: Quality of Life in Head and Neck Cancer 6th International Workshop; 2008; Liverpool, England.

19. Head and Neck Cancers Patient Views and Experiences. Oxford, England: The National Cancer Alliance; 2002.

20. Mesters I, van den Borne B, De Boer M, Pruyn, J. Measuring information needs among cancer patients. Patient Educ Couns. 2001;43:253-262.

21. Edwards, D. Head and neck cancer services: views of patients, their families and professionals. Br J Oral Maxillofac Surg. 1998;36:99102.

22. Vartanian JG, Carvalho AL, Toyota J, Kowalski IS, Kowalski LP. Socioeconomic effects of and risk factors for disability in long-term survivors of head and neck cancer. Arch Otolaryngol Head Neck Surg. 2006;132:32-35.

23. MacInnes T, Parekh A, Kenway P. London's Poverty Profile 2011. New Policy Institute. http://www.londonspovertyprofile.org.uk/ downloads/povertyreport2011-web.pdf. Published 2011. Accessed July 12, 2015. 